\title{
Determination of Serum Homocysteine and Hypersensitive C-reactive Protein and Their Correlation with Premature Coronary Heart Disease
}

\author{
Songsen Li, Guangjie Pan, Huili Chen, Xiaohua Niu \\ Department of Cardiology, Luoyang Center Hospital Affiliated to Zhengzhou University, Luoyang, China
}

\section{ABSTRACT}

Background: This study aims to investigate the correlation between premature coronary heart disease (pCHD) and both serum homocysteine (Hcy) and hypersensitive C-reactive protein (hs-CRP).

Methods: A total of 170 patients with pCHD were enrolled in this study from June 2014 to April 2016 (including 52 patients with stable angina pectoris [SAP], 70 patients with unstable angina pectoris [UAP], and 48 patients with acute myocardial infarction [AMI]), together with 105 healthy controls $(\mathrm{CON})$ selected at the same period, to observe the changes of Hcy and hs-CRP in CHD patients and those with different types of CHD.

Results: The levels of serum Hcy and hs-CRP in group pCHD were significantly higher than in group CON $(P<$ $.05)$. The levels of Hcy and hs-CRP in group AMI were significantly higher than in group UAP and group SAP $(P<.05)$. The changes of serum Hcy and hs-CRP were significantly higher in patients with multi-vascular lesions and dual-vascular lesions than in those with single-vascular lesion $(P<.05)$.

Conclusion: The levels of serum Hcy and hs-CRP in CHD patients are positively correlated with the severity of $\mathrm{CHD}$, which increase with the increase of lesion count. The combined detection of Hcy and hs-CRP can initially reflect the severity of coronary artery, thus better guiding treatment and predicting prognosis.

\section{INTRODUCTION}

Coronary atherosclerotic heart disease (CHD) has increased in recent years, and its onset age tends toward younger people. Atherosclerosis and plaque rupture are its pathological basis. Studies have shown that homocysteine (Hcy) is an independent risk factor for CHD, which has significant atherogenic effects [Schroecksnadel 2010]. Chronic inflammatory response is also involved in the progression of atherosclerosis, and hypersensitive C-reactive protein (hs-CRP) is an important inflammatory factor for atherosclerosis [Devaraj 2009; Libby 2010; Weber 2011]. Animal

Received November 20, 2018; accepted March 11, 2019.

Correspondence: Xiaohua Niu, Department of Cardiology, Luoyang Center Hospital Affiliated to Zhengzhou University, Luoyang 471000, China; +8613938801812; fax: +86-379-63892228 (e-mail: shouyanzhang@126.com). experiments have shown that Hcy upregulates the expression of hs-CRP in the vascular smooth muscles by oxidative stress and cell proliferation. Hcy and CRP have interactions in atherosclerosis [Pang 2014; Pang 2015]. Clinical trials have shown that the levels of CRP and IL-6 in patients with high hcyemia are significantly higher than in healthy people [Holven 2006], and Hcy is positively correlated with such inflammatory indexes as CRP and complement $4[\mathrm{Li}$ 2015]. Clinical studies have shown that the combination of Hcy and hs-CRP can better predict the clinical prognosis of acute cerebral infarction than hs-CRP alone [Gong 2013]. Cross-sectional studies have shown that Hcy and hs-CRP can better predict the progression and prognosis of perforator artery (BAD) cerebral infarction [Men 2013]. However, the conclusion of combining Hcy and hs-CR in the same CHD patient is still not entirely consistent. In this study, we analyzed the changes of Hcy and hs-CRP in patients with pCHD and healthy controls, and compared the concentrations of Hcy and hs-CRP in patients with different severities of CHD, aiming to explore the correlation of serum Hcy and hs-CRP with pCHD and to evaluate their clinical values in predicting the severity of CHD.

\section{MATERIALS AND METHODS}

\section{Subjects}

A total of 170 patients with CHD were enrolled from June 2014 to April 2016, including 52 patients with stable angina pectoris (SAP), 70 patients with unstable angina pectoris (UAP), and 48 patients with acute myocardial infarction (AMI). A total of 105 healthy subjects were selected as the control group during the same period. Exclusion criteria were malignant tumors, autoimmune diseases, acute/chronic infections, trauma, liver/brain/kidney/endocrine/other cardiovascular disease. Subjects were not given folic acid, vitamin B, and drugs that might affect the metabolism of Hcy, such as methotrexate, phenytoin, and theophylline within 2 weeks before admission. The CHD patients were subdivided into group Single (55 patients with single-vascular lesion), group Double (53 patients with dual-vascular lesions), and group Multiple (62 patients with multi-vascular lesions). The comparison of general clinical data showed no significant difference in the age and smoking history between the two groups $(P>.05)$, but the two groups showed statistical significance in diabetes and triglycerides $(P<.05)$ (Table 1$)$. This study was conducted in accordance with the Declaration of Helsinki. 
Table 1. Comparison of Clinical Data in pCHD and CON Groups

\begin{tabular}{lcc}
\hline & $\mathrm{pCHD}(\mathrm{n}=170)$ & $\mathrm{CON}(\mathrm{n}=105)$ \\
\hline $\mathrm{M} / \mathrm{F}$ & $92 / 78$ & $56 / 49$ \\
Age, $\mathrm{y}$ & $56.1 \pm 6.24$ & $55.3 \pm 6.83$ \\
Hypertension & $245(72.06)$ & $166(69.17)$ \\
Diabetes & $5.92 \pm 1.26^{*}$ & $4.14 \pm 1.18^{*}$ \\
Smoking & $83(48.82)$ & $33(31.42)$ \\
BMl, $\mathrm{kg} / \mathrm{m}^{2}$ & $25.12 \pm 3.02$ & $24.99 \pm 2.66$ \\
TC, $\mathrm{mmol} / \mathrm{L}$ & $4.58 \pm 0.76$ & $4.52 \pm 0.48$ \\
$\mathrm{LDL}-\mathrm{C}, \mathrm{mmol} / \mathrm{L}$ & $2.94 \pm 0.83$ & $2.80 \pm 0.92$ \\
$\mathrm{HDL}-\mathrm{C}, \mathrm{mmol} / \mathrm{L}$ & $0.95 \pm 0.26$ & $0.97 \pm 0.32$ \\
TG, $\mathrm{mmol} / \mathrm{L}$ & $1.86 \pm 1.23^{*}$ & $1.27 \pm 0.67$ \\
\hline
\end{tabular}

*Compared with CON, $P<.05$.

BMI indicates body mass index; TC, total cholesterol; LDL-C, low density lipoprotein cholesterol; HDL-C, high density lipoprotein cholesterol; TG, triglyceride.

This study was conducted with approval from the Ethics Committee of Zhengzhou University. Written informed consent was obtained from all participants.

\section{Determination of $\mathrm{Hcy}$ and $\mathrm{bs}-\mathrm{CRP}$}

3-5 mL of fasting venous blood was collected from each subject, followed by $10-\mathrm{min}$ centrifugation at $3000 \mathrm{r} / \mathrm{min}$ to separate the serum, and then completion of detection was done within $2 \mathrm{~h}$. Hcy was tested using the cyclophorase method, and hs-CRP was tested by immunoturbidimetry; all the reagents, calibration fluid, and quality control standards were provided by Beijing Reegen BioTech, and all tests were performed using one Olympus AU2700 automatic biochemical analyzer (Japan), strictly in accordance with the operating instructions or standard operating procedures.

\section{Evaluation of Severity of CHD Lesions}

Coronary angiography was performed using the Judkins method [Plank 2016]. The coronary artery lesions were observed from the conventional projection view. The angiographic images were analyzed by two experienced cardiovascular physicians for evaluating stenosis in the left main branch, left anterior descending branch, left circumflex branch, right coronary artery, and their primary branches. Angiographic stenosis $\geq 50 \%$ in one, two, or three major coronary arteries was defined as single, dual, or triple lesions.

\section{Statistical Analysis}

SPSS 17.0 software was used for statistical analysis; the measurement data were expressed as mean \pm standard deviation and tested using Student $t$ test; count data were tested using the Pearson chi-square test, with $P<.05$ considered statistically significant.
Table 2. Comparison of Hcy and hs-CRP Levels in PCHD and CON Groups

\begin{tabular}{lccc}
\hline Groups & $n$ & Hcy $($ ummol $/ \mathrm{L})$ & hs-CRP $(\mathrm{ml} / \mathrm{L})$ \\
\hline PCHD & 170 & $22.63 \pm 5.18^{*}$ & $22.96 \pm 4.73^{*}$ \\
CON & 105 & $8.11 \pm 2.42$ & $2.33 \pm 0.49$ \\
\hline
\end{tabular}

*Compared with CON, $P<.05$.

\section{RESULTS}

Comparison of Hcy and hs-CRP in Group pCHD and Group CON

The Hcy and hs-CRP levels in group pCHD were significantly higher than in group $\operatorname{CON}(P<.01$; Table 2$)$.

\section{Comparison of Observation Indexes among Groups with Different $C H D$}

The Hcy and hs-CRP levels in group AMI and group UAP were significantly higher than in group $\operatorname{SAP}(P<.05$; Table 3$)$.

\section{Correlation of Severity of CHD with Levels of Hcy and bs-CRP}

Compared with group CON, the Hcy and hs-CRP levels in patients with single, dual, or triple CHD lesions were significantly higher $(P<.05)$. The comparison among the groups with different CHD showed that the Hcy and hs-CRP levels were significantly higher in patients with multi-vascular lesions and dual-vascular lesions than in those with singlevascular lesion $(P<.05$; Table 4$)$.

\section{DISCUSSION}

This study shows that the Hcy and hs-CRP levels in group pCHD were significantly higher than in group CON; the levels of Hcy and hs-CRP in group AMI and UAP were higher than in group SAP; and the levels of Hcy and hs-CRP in patients with multi- or dual-vascular lesions were significantly higher than in those with single-vascular lesion, indicating that Hcy is related to the severity of CHD. Clinical studies have shown that serum markers such as Hcy are associated with the severity of CHD lesions [Feng 2016]. Another study showed that the Hcy level in $79.1 \%$ of CHD patients was higher than $15.0 \mu \mathrm{mol} / \mathrm{L}$, but that in only $5 \%$ of patients with non-coronary heart disease was it above $15.0 \mu \mathrm{mol} / \mathrm{L}$. Moreover, $66 \%$ of patients with SAP, $81.9 \%$ of patients with UAP, and $93.15 \%$ of patients with AMI showed Hcy higher than $15.0 \mu \mathrm{mol} / \mathrm{L}$ [Liu 2015]. This may be due to the fact that the higher the Hcy level, the more severe the oxidative stress reaction-resulted vascular endothelial damage and dysfunction [Steed 2011].

CRP is a simple and reliable predictor of CHD, and epidemiological meta-analyses have shown that CRP $>3.0 \mathrm{mg} / \mathrm{dL}$ increases the risk of CHD by 1.6 times than that of $<1.0 \mathrm{mg} /$ 
Table 3. Comparison of Hcy and hs-CRP Levels among Groups with Different CHD

\begin{tabular}{lccc}
\hline Group & $\mathrm{n}$ & Hcy $($ ummol/L) & hs-CRP $(\mathrm{ml} / \mathrm{L})$ \\
\hline SAP & 52 & $14.42 \pm 3.76$ & $9.32 \pm 2.78$ \\
UAP & 70 & $18.32 \pm 4.17^{*}$ & $16.54 \pm 4.27^{*}$ \\
AMI & 48 & $29.16 \pm 6.94^{*}$ & $29.96 \pm 8.16^{*}$ \\
\hline
\end{tabular}

*Compared with SAP, $P<.05$.

dL [Buckley 2009]. CRP is significantly elevated in patients with severe UAP and can suggest poor prognosis [Liuzzo 1994; Strang 2014]. Significantly elevated CRP after myocardial infarction is associated with poor prognosis, including heart failure, cardiac death, or heart rupture [Pietilä 1996; Suleiman 2003; Suleiman 2006]. Recent studies have shown that CRP genotypes are related to the incidence of CHD [Hernández-Díaz 2015]. This study shows that CRP is associated with the severity of CHD, and that patients with AMI or UAP have significantly increased CRP levels compared to those with SAP; and CRP levels in patients with triple- and dual-vascular CHD are significantly higher than in those with single-vascular lesion.

Hcy and CRP are both predictors of CHD, and the data in this study show that the combination of Hcy and CRP can more accurately reflect the severity of CHD and coronary artery disease. The levels of Hcy and CRP in group AMI were significantly higher than in those in group SAP, and CRP levels in patients with triple- and dual-vascular CHD were significantly higher than in those with SAP.

\section{REFERENCES}

Buckley DI, Fu R, Freeman M, et al. 2009. C-reactive protein as a risk factor for coronary heart disease: a systematic review and meta-analyses for the U.S. Preventive Services Task Force. Ann Intern Med 151:483-95.

Devaraj S, Singh U, Jialal I. 2009. The evolving role of C-reactive protein in atherothrombosis. Clin Chem 55:229-38.

Feng L, Nian S, Zhang S, et al. 2016. The associations between serum biomarkers and stenosis of the coronary arteries. Oncotarget 7:39231-40.

Gong X, Zou X, Liu L, et al. 2013. Prognostic value of inflammatory mediators in 1-year outcome of acute ischemic stroke with middle cerebral artery stenosis. Mediators Inflamm 2013:850714.

Hernández-Díaz Y, Tovilla-Zárate CA, Juárez-Rojop I, et al. 2015. The role of gene variants of the inflammatory markers CRP and TNF- $\alpha$ in cardiovascular heart disease: systematic review and meta-analysis. Int $\mathrm{J}$ Clin Exp Med 8:11958-84.

Holven KB, Aukrust P, Retterstol K, et al. 2006. Increased levels of C-reactive protein and interleukin-6 in hyperhomocysteinemic subjects. Scand J Clin Lab Invest 66:45-54.

Li T, Chen Y, Li J, et al. 2015. Serum homocysteine concentration is significantly associated with inflammatory/immune factors. PLoS One 10:e0138099.
Table 4. Comparison of Hcy and hs-CRP Levels among Groups with Different CHD

\begin{tabular}{lccc}
\hline Groups & $\mathrm{n}$ & $\mathrm{HCY}(\mathrm{ummol} / \mathrm{L})$ & $\mathrm{hs}-\mathrm{CRP}(\mathrm{ml} / \mathrm{L})$ \\
\hline CON & 105 & $8.11 \pm 2.42^{*}$ & $2.33 \pm 0.49 *$ \\
Single lesion & 55 & $21.26 \pm 4.38 \dagger$ & $10.87 \pm 3.26 \dagger$ \\
Dual lesions & 53 & $26.87 \pm 5.96$ & $21.6 \pm 4.58$ \\
Triple lesions & 62 & $27.02 \pm 4.17$ & $25.96 \pm 7.46$ \\
\hline
\end{tabular}

*Compared with Group CON, $P<.05$.

†Compared with Group Single, $P<.05$.

Libby P, Okamoto Y, Rocha VZ, et al. 2010. Inflammation in atherosclerosis: transition from theory to practice. Circ J 74:213-20.

Liu C, Yang Y, Peng D, et al. 2015. Hyperhomocysteinemia as a metabolic disorder parameter is independently associated with the severity of coronary heart disease. Saudi Med J 36:839-46.

Liuzzo G, Biasucci LM, Gallimore JR, et al. 1994. The prognostic value of C-reactive protein and serum amyloid A protein in severe unstable angina. N Engl J Med 331:417-24.

Men X, Li J, Zhang B, et al. 2013. Homocysteine and C-reactive protein associated with progression and prognosis of intracranial branch atheromatous disease. PLoS One 8:e73030.

Pang X, Liu J, Zhao J, et al. 2014. Homocysteine induces the expression of C-reactive protein via NMDAr-ROS-MAPK-NF- B signal pathway in rat vascular smooth muscle cells. Atherosclerosis 236:73-81.

Pang X, Liu J, Li Y, et al. 2015. Emodin inhibits homocysteine-induced $\mathrm{C}$-reactive protein generation in vascular smooth muscle cells by regulating PPAR $\gamma$ expression and ROS-ERK1/2/p38 signal pathway. PLoS One 10:e0131295.

Pietilä KO, Harmoinen AP, Jokiniitty J, et al. 1996. Serum C-reactive protein concentration in acute myocardial infarction and its relationship to mortality during 24 months of follow-up in patients under thrombolytic treatment. Eur Heart J 17:1345-9.

Plank F, Burghard P, Friedrich G, et al. 2016. Quantitative coronary CT angiography: absolute lumen sizing rather than \% stenosis predicts hemodynamically relevant stenosis. Eur Radiol 26:3781-9.

Schroecksnadel K, Grammer TB, Boehm BO, et al. 2010. Total homocysteine in patients with angiographic coronary artery disease correlates with inflammation markers. Thromb Haemost 103:926-35.

Steed MM, Tyagi SC. 2011. Mechanisms of cardiovascular remodeling in hyperhomocysteinemia. Antioxid Redox Signal 15:1927-43.

Strang F, Schunkert H. 2014. C-reactive protein and coronary heart disease: all said—is not it? Mediators Inflamm 2014:757123.

Suleiman M, Aronson D, Reisner SA, et al. 2003. Admission C-reactive protein levels and 30-day mortality in patients with acute myocardial infarction. Am J Med 115:695-701.

Suleiman M, Khatib R, Agmon Y, et al. 2006. Early inflammation and risk of long-term development of heart failure and mortality in survivors of acute myocardial infarction predictive role of C-reactive protein. J Am Coll Cardiol 47:962-8.

Weber C, Noels H. 2011. Atherosclerosis: current pathogenesis and therapeutic options. Nat Med 17:1410-22. 